\title{
DOMINATION AND ERGODICITY FOR POSITIVE SEMIGROUPS
}

\author{
W. ARENDT AND C. J. K. BATTY
}

(Communicated by Palle E. T. Jorgensen)

\begin{abstract}
In this note we study the conditions under which ergodicity is preserved by domination. As an application, the criterion for almost periodicity given by Ljubich-Phong [9] (see also Batty-Phong [4]) can be simplified for positive semigroups.
\end{abstract}

\section{ERGODICITY AND DOMINATION}

Let $T=(T(t))_{t \geq 0}$ be a positive $C_{0}$-semigroup on a Banach lattice $E$ with generator $A$. As usual, we say that $T$ is mean-ergodic (or $C$-ergodic) if $C-$ $\lim _{t \rightarrow \infty} T(t):=\lim _{t \rightarrow \infty} \frac{1}{t} \int_{0}^{t} T(s) d s$ exists strongly. Due to the positivity of $T$, this is equivalent to the following two conditions (see [3]):

$$
(0, \infty) \subset \rho(A) \text { and } \sup _{0<\lambda \leq 1}\|\lambda R(\lambda, A)\|<\infty
$$

and

$N(A)$ separates $N\left(A^{\prime}\right)$ (i.e. for all $0 \neq \varphi \in N\left(A^{\prime}\right)$ there exists $x \in N(A)$ such that $\langle x, \varphi\rangle \neq 0)$.

Here $N(A):=\{x \in D(A): A x=0\}$ denotes the kernel of $A$ and $N\left(A^{\prime}\right)$ the kernel of $A^{\prime}$.

We are going to show that ergodicity is preserved by domination if additional conditions (order continuous norm or irreducibility) are satisfied. Without any additional assumptions, this is false as is shown by examples in $\S 3$.

Theorem 1.1. Assume that $E$ has order continuous norm. Let $S$ and $T$ be $C_{0}$-semigroups on $E$ such that

$$
|S(t) x| \leq T(t)|x| \quad(t \geq 0, x \in E) .
$$

If $T$ is mean-ergodic, then $S$ is also mean-ergodic.

Proof. Let $x \in E$. Since $T$ is mean-ergodic, the set $H_{x}:=\left\{\frac{1}{t} \int_{0}^{t} T(s)|x| d s\right.$ : $t>0\} \subset E_{+}$is relatively weakly compact.

Since $E$ has order continuous norm, $E$ is an ideal in $E^{\prime \prime}$ and the solid hull so $\left(H_{x}\right):=\left\{y \in E:|y| \leq u\right.$ for some $\left.u \in H_{x}\right\}$ is relatively weakly compact

Received by the editors September 20, 1990.

1991 Mathematics Subject Classification. Primary 47A35, 47006. 
as well (cf. $[1,13.8])$. Hence $\left\{\frac{1}{t} \int_{0}^{t} S(s) x d s\right\} \subset$ so $\left(H_{x}\right)$ is relatively weakly compact for all $x \in E$, and so $S$ is mean-ergodic [7].

Corollary 1.2. If $E$ is a complex Banach lattice with order-continuous norm and $T$ is a positive, mean-ergodic $C_{0}$-semigroup, then $\left(e^{-i \alpha t} T(t)\right)_{t \geq 0}$ is also meanergodic for all real $\alpha$.

Theorem 1.3. Let $T$ be a $C_{0^{-}}$-semigroup with generator $A$ and $S$ be $a C_{0^{-}}$ semigroup with generator $B$ such that

$$
0 \leq S(t) \leq T(t) \quad(t \geq 0) .
$$

Assume that $T$ is irreducible and mean-ergodic. If $N\left(B^{\prime}\right) \neq\{0\}$, then $S(t)=$ $T(t) \quad(t \geq 0)$.

Concerning the notion of irreducibility we refer to [5] or [10].

Proof. It follows from the domination property that $(0, \infty) \subset \rho(B)$ (cf. [10, C-II, Lemma 4.10]). Assume there exists $0 \neq \psi \in E^{\prime}$ such that $S(t)^{\prime} \psi=\psi$ $(t \geq 0)$. So $S(t)^{\prime}|\psi| \geq|\psi|(t \geq 0)$. Consequently $S(s+t)^{\prime}|\psi| \geq S(s)^{\prime}|\psi|$ $(s, t \geq 0)$; i.e. $S(t)^{\prime}|\psi|$ is increasing. Hence $\lambda R(\lambda, B)^{\prime}|\psi|=\int_{0}^{\infty} \lambda e^{-\lambda t} S(t)^{\prime}|\psi| d t$ is increasing in $\lambda$. But $\sup _{1 \geq \lambda>0}\left\|\lambda R(\lambda, B)^{\prime}\right\| \leq \sup _{1 \geq \lambda>0}\|\lambda R(\lambda, A)\|<\infty$, since $T$ is mean-ergodic. Then $\varphi:=\sup _{0<\lambda \leq 1} \lambda R(\lambda, B)^{\prime}|\psi| \in N\left(B^{\prime}\right) \cap E_{+}, \varphi \neq 0$. Thus $0 \leq \varphi=S(t)^{\prime} \varphi \leq T(t)^{\prime} \varphi$. By the same argument as before $\mu:=$ $\sup _{0<\lambda<1} \lambda R(\lambda, A)^{\prime} \varphi \in N\left(A^{\prime}\right), \mu \geq 0, \mu \neq 0$. It follows that $\mu \gg 0$ (i.e. $\langle x, \mu\rangle>0$ for all $\left.x \in E_{+}, x \neq 0\right)$ since $T$ is irreducible. Since $T$ is meanergodic, it follows that $N(A) \neq 0$. Let $v \in N(A), v \neq 0$. Then $T(t)|v|-|v| \geq 0$ and $\langle T(t)|v|-|v|, \mu\rangle=0$. Hence $u:=|v| \in N(A), u \neq 0$. Since $T$ is irreducible, it follows that $u$ is a quasi-interior point. Now $T(t)^{\prime} \varphi-\varphi \geq 0$ and $\left\langle u, T(t)^{\prime} \varphi-\varphi\right\rangle=0$. Hence $T(t)^{\prime} \varphi=\varphi, t \geq 0$. It follows that $\varphi \gg 0$ by the irreducibility of $T$.

We have shown that $0 \ll \varphi=S(t)^{\prime} \varphi=T(t)^{\prime} \varphi \quad(t \geq 0)$. Let $x \in E_{+}$. Then $T(t) x-S(t) x \geq 0$ and $\langle T(t) x-S(t) x, \varphi\rangle=0$. Hence $T(t) x=S(t) x \quad(t \geq 0)$. This shows that $T(t)=S(t) \quad(t \geq 0)$.

Remark. By a slight alteration of the proof of Theorem 1.3, one obtains the following.

Assume that $E$ is an order complete real or complex Banach lattice. Let $T$ and $S$ be two $C_{0}$-semigroups on $E$ with generators $A$ and $B$, respectively, such that $|S(t) x| \leq T(t) x$ for all $x \in E_{+}, t \geq 0$. If $T$ is irreducible and mean-ergodic and if $N\left(B^{\prime}\right) \neq\{0\}$, then $|S(t)|=T(t) \quad(t \geq 0)$.

Corollary 1.4. Let $S$ and $T$ be positive $C_{0}$-semigroups such that $0 \leq S(t) \leq$ $T(t) \quad(t \geq 0)$. Assume that $T$ is irreducible and mean-ergodic. Then $S$ is mean-ergodic and $C-\lim _{t \rightarrow \infty} S(t)=0$ strongly if $S \neq T$.

Proof. By Proposition 1.3, $S=T$ if $N\left(B^{\prime}\right) \neq\{0\}$. Assume that $N\left(B^{\prime}\right)=\{0\}$. Then $N(B)$ separates $N\left(B^{\prime}\right)$. Hence $S$ is mean-ergodic and it follows from the general theory that $C-\lim _{t \rightarrow \infty} S(t)=0$.

\section{ALMOST PERIODIC SEMIGROUPS}

Let $\{T(t): t \geq 0\}$ be a bounded $C_{0}$-semigroup on a Banach space $E$ with generator $A$, and suppose that $\sigma(A) \cap i \mathbb{R}$ is countable. It has been shown that 
$T$ is stable if (and only if) $R \sigma(A) \cap i \mathbf{R}$ is empty [2,8], and $T$ is almost periodic if (and only if) $E \sigma(A) \cap i \mathbf{R}$ is empty [4, 9]. Here

$$
\begin{aligned}
R \sigma(A) & =P \sigma\left(A^{\prime}\right)=\{\lambda \in \mathbb{C}: R(\lambda-A) \text { is not dense in } E\} \text { and } \\
E \sigma(A) & =\{\lambda \in \mathbb{C}: R(\lambda-A)+N(\lambda-A) \text { is not dense in } E\} \\
& =\left\{\lambda \in \mathbb{C}: N(\lambda-A) \text { does not separate } N\left(\lambda-A^{\prime}\right)\right\},
\end{aligned}
$$

where $R(\lambda-A)$ denotes the range of $(\lambda-A)$. Thus

$$
\begin{gathered}
E \sigma(A) \cap i \mathbb{R}=\left\{i \alpha: \alpha \in \mathbb{R}, e^{-i \alpha t} T(t) \text { is not mean-ergodic }\right\}, \\
P \sigma\left(A^{\prime}\right)=P \sigma(A) \cup E \sigma(A) .
\end{gathered}
$$

Now suppose that $E$ is a Banach lattice, and $T(t) \geq 0$. If $T^{\prime}(t) \varphi=e^{i \alpha t} \varphi$, then $t \mapsto T^{\prime}(t)|\varphi|$ is bounded and increasing, and its weak ${ }^{*}$ limit is a fixed point of $T^{\prime}$. Thus if $R \sigma(A) \cap i \mathbb{R}$ is nonempty, then $O \in R \sigma(A)$. Hence, if $\sigma(A) \cap i \mathbb{R}$ is countable and $O \notin R \sigma(A)$, then $T$ is stable. In fact, for positive semigroups, $\operatorname{R\sigma }(A) \cap i \mathbb{R}$ is always cyclic; assuming $s(A):=\sup \{\operatorname{Re} \lambda: \lambda \in \sigma(A)\}=0$, this means that $i \alpha \in R \sigma(A)$ implies $i n \alpha \in R \sigma(A)$ for all $n \in \mathbb{Z}$ (where $\alpha \in \mathbb{R}$ ), see proof of [10, C-III, Corollary 4.3].

It is a natural to question whether there are corresponding results for $E \sigma(A) \cap$ $i \mathbb{R}$ and for almost periodicity. We show that there are such results if $E$ has order-continuous norm, but not in general, even for $C_{0}$-groups of lattice isomorphisms.

Remark. If the Banach space is reflexive and $\sup _{0<\lambda \leq 1}\|\lambda R(\lambda, A)\|<\infty$ (e.g., if $T$ is bounded), then $E \sigma(A)$ is empty.

First we note as an immediate consequence of Corollary 1.2 and [4, Theorem 8] (or [9]) the following.

Theorem 2.1. If $E$ has order-continuous norm and $T$ is a bounded, positive, mean-ergodic $C_{0}$-semigroup on $E$, and $\sigma(A) \cap i \mathbb{R}$ is countable, then $T$ is almost periodic.

Theorem 2.2. Let $T$ be a bounded, irreducible, positive $C_{0}$-semigroup with generator $A$ and assume that $E$ has order-continuous norm. Then $E \sigma(A)$ is cyclic. Proof. Let $\eta \in \mathbb{R}$ such that $i \eta \in E \sigma(A)$. We have to show that in $\eta \in E \sigma(A)$ for all $n \in \mathbb{Z}$. It follows from Corollary 1.2 that $O \in E \sigma(A)$. Hence $N\left(A^{\prime}\right) \neq$ \{0\} (otherwise $N(A)$ separates $N\left(A^{\prime}\right)$ ). By the proof of Theorem 1.3, there exists $0 \neq \psi \in N\left(A^{\prime}\right)_{+}$. Since $T$ is irreducible, it follows that $\psi \gg 0$. As a consequence, $N(A)=\{0\}$ (in fact, otherwise there exists a quasi-interior point $u \in N(A)$ (by the proof of Theorem 1.3). Since order intervals are weakly compact, it follows that $\left\{\frac{1}{t} \int_{0}^{t} T(s) x d s: t>0\right\}$ is relatively weakly compact whenever $|x| \leq m \cdot u$ for some $m \in \mathbb{N}$. The set of such vectors $x$ is dense, so $T$ is mean-ergodic, contradicting $O \in E \sigma(A))$. It follows from [10, C-III, Theorem 3.8] that $P \sigma(A) \cap i \mathbb{R}=\varnothing$. Hence $i \eta \in E \sigma(A) \backslash P \sigma(A) \subset R \sigma(A)$. So the claim follows since $R \sigma(A)$ is cyclic (by the proof of [10, C-III, Corollary 4.3]).

\section{CountereXAmples}

The first example shows that Theorems 1.1 and 1.3 may fail if the norm is not order-continuous or $T$ is not irreducible. 
Example 3.1. Let $E=C(\Omega)$ where $\Omega$ is compact, and let $\lambda: \Omega \rightarrow[0, \infty)$ be continuous such that $\lambda^{-1}(0)$ is not open in $\Omega$. Define $T$ on $E$ by $T(t)=I$ $(t \geq 0)$ and $S$ by $S(t) f=e^{-t \lambda} f$. Then $\left(S(t) 1_{\Omega}\right)(\omega) \rightarrow 1 \quad(t \rightarrow \infty)$ if $\lambda(\omega)=0$ and $\left(S(t) 1_{\Omega}\right)(\omega) \rightarrow 0$ if $\lambda(\omega)>0$. So $S$ is not mean ergodic.

Example 3.2. This example shows that the Corollary 1.2 and Theorem 2.1 may fail if the norm is not order-continuous, even if $T$ is a $C_{0}$-group of isometric lattice-isomorphisms. Let $K$ be a compact subset of $[0, \infty), \Omega=\left\{r e^{i \theta}: r \in\right.$ $K, 0 \leq \theta<2 \pi\}$ and $E=C(\Omega)$. Define

$$
T(t) f\left(r e^{i \theta}\right)=f\left(r e^{i(\theta+t r)}\right) .
$$

For real $x$, let

$$
\begin{gathered}
K_{\alpha}=\{r \in K: \alpha \in r \mathbb{Z}\}, \\
f_{\alpha}(r)=\frac{1}{2 \pi} \int_{0}^{2 \pi} e^{-i \alpha \theta / r} f\left(r e^{i \theta}\right) d \theta \quad\left(f \in E, r \in K_{\alpha}, r>0\right), \\
f_{0}(0)=0 .
\end{gathered}
$$

Now $N(A-i \alpha)$ consists of all functions of the form $f\left(r e^{i \theta}\right)=g(r) e^{i \alpha \theta / r}$, where $g \in C(K), g(r)=0$ if $r \notin K_{\alpha}$. Also $N\left(A^{\prime}-i \alpha\right)$ consists of all functionals of the form

$$
f \mapsto \int_{K_{\alpha}} f_{\alpha}(r) d \mu(r),
$$

where $\mu$ is any measure on $K_{\alpha}$. In particular, for nonzero $\alpha, i \alpha \notin E \sigma(A)$ if and only if, whenever $\alpha / n \in K$ for a nonzero integer $n$, then $\alpha / n$ is isolated in $K$. But $T$ is mean-ergodic, as may be seen directly or by observing that $N(A)$ separates $N\left(A^{\prime}\right)$. Moreover,

$$
\sigma(A)=\{\text { inr }: n \in \mathbb{Z}, r \in K\}^{-} .
$$

In particular, if $K=[0,1]$, so $\Omega$ is the unit disc, then $e^{-i \alpha t} T(t)$ is meanergodic if and only if $\alpha=0$. If $K=\left\{1+2^{-m}: m \geq 1\right\} \cup\{1\}$, then $\sigma(A)=$ $\left\{n\left(1+2^{-m}\right): m \geq 1, n \in \mathbb{Z}\right\} \cup \mathbb{Z}$, so $\sigma(A)$ is countable and $T$ is mean-ergodic. However, $T$ is not almost periodic, since each eigenfunction is constant on the circle $r=1$.

\section{REFERENCES}

1. C. D. Aliprantis and O. Burkinshaw, Positive operators, Academic Press, London, 1985.

2. W. Arendt and C. J. K. Batty, Tauberian theorems and stability of one-parameter semigroups, Trans. Amer. Math. Soc. 306 (1988), 837-852.

3. W. Arendt and J. Prüss, Vector-valued Tauberian theorems and asymptotic behavior of linear Volterra equations, SIAM J. Math. Anal. (to appear).

4. C. J. K. Batty and Vu Quoc Phong, Stability of individual elements under one-parameter semigroups, Trans. Amer. Math. Soc. (to appear).

5. C. J. K. Batty and D. Robinson, Positive one-parameter semigroups on ordered spaces, Acta Appl. Math. 2 (1984), 221-296.

6. R. Derndinger, R. Nagel, and G. Palm, Ergodic theory in the perspective of functional analysis, Tübingen, 1987.

7. U. Krengel, Ergodic theorems, De Gruyter, Berlin, 1985.

8. Yu I. Ljubich and Vu Quoc Phong, Asymptotic stability of linear differential equations on Banach spaces, Studia Math. 88 (1988), 37-42. 
9. __ A spectral criterion for almost periodicity of one-parameter semigroups, Teor. Funktsii. Funktsional. Anal. i Prilozhen. 47 (1987), 36-41. (Russian)

10. R. Nagel, One-parameter semigroups of positive operators, Lecture Notes in Math., vol. 1184, Springer-Verlag, Berlin, 1986.

Equipe de Mathématiques, U.A. CNRS 741, Universite de Franche-Comte, 25030 Besançon Cedex France

St. JohN's COLlege, OXford OX1 3JP ENGLAND 